\title{
Tri-bimaximal Neutrino Mixing and Flavor-dependent Resonant Leptogenesis
}

\author{
Zhi-zhong Xing* and Shun Zhou ${ }^{*}$ \\ Institute of High Energy Physics, Chinese Academy of Sciences, \\ P.O. Box 918(4), Beijing 100049, China
}

\begin{abstract}
We propose a particularly economical neutrino mass model, in which there are only two righthanded Majorana neutrinos of $\mathcal{O}(1) \mathrm{TeV}$ and their masses are highly degenerate. Its novel Yukawacoupling texture together with the seesaw mechanism allows us to achieve the normal neutrino mass hierarchy with $m_{1}=0$ and a nearly tri-bimaximal neutrino mixing pattern with the maximal CPviolating phase: $\theta_{23}=\pi / 4,|\delta|=\pi / 2$ and $\sin ^{2} \theta_{12}=\left(1-2 \tan ^{2} \theta_{13}\right) / 3$. One may also obtain the inverted neutrino mass hierarchy with $m_{3}=0$ and the corresponding neutrino mixing pattern with $\theta_{23}=\pi / 4$ and $\theta_{13}=\delta=0$. In both cases, it is possible to interpret the cosmological baryon number asymmetry $\eta_{\mathrm{B}} \approx 6.1 \times 10^{-10}$ through the resonant leptogenesis mechanism. We demonstrate the significance of flavor-dependent effects in this leptogenesis scenario: they can either flip the sign of the flavor-independent prediction for $\eta_{\mathrm{B}}$ in the $m_{1}=0$ case or magnify the magnitude of the flavor-independent prediction for $\eta_{\mathrm{B}}$ about 50 times in the $m_{3}=0$ case.
\end{abstract}

PACS numbers: 11.30.Fs, 14.60.Pq, 14.60.St

*Electronic address: xingzz@mail.ihep.ac.cn

${ }^{\dagger}$ Electronic address: zhoush@mail.ihep.ac.cn 


\section{INTRODUCTION}

The recent solar [1], atmospheric [2], reactor [3] and accelerator [4] neutrino oscillation experiments have provided us with very robust evidence that neutrinos are massive and their flavor mixing involves two large angles $\left(\theta_{12} \sim 34^{\circ}\right.$ and $\left.\theta_{23} \sim 45^{\circ}\right)$ and one small angle $\left(\theta_{13}<10^{\circ}\right)$. These important results indicate that the minimal standard electroweak model, in which neutrinos are massless Weyl particles, is incomplete. A very economical extension of the standard model, the so-called minimal seesaw model [5], is to introduce two heavy righthanded Majorana neutrinos $\left(N_{1}\right.$ and $\left.N_{2}\right)$ but preserve its $S U(2)_{\mathrm{L}} \times U(1)_{\mathrm{Y}}$ gauge symmetry. In this case, the Lagrangian relevant for lepton masses can be written as

$$
-\mathcal{L}_{\text {lepton }}=\overline{l_{\mathrm{L}}} Y_{l} E H+\overline{l_{\mathrm{L}}} Y_{\nu} N H^{\mathrm{c}}+\frac{1}{2} \overline{N^{\mathrm{c}}} M_{\mathrm{R}} N+\text { h.c. }
$$

where $l_{\mathrm{L}}$ denotes the left-handed lepton doublet, $H$ is the Higgs-boson weak isodoublet $\left(H^{\mathrm{c}} \equiv\right.$ $\left.i \sigma_{2} H^{*}\right), E$ and $N$ stand respectively for the right-handed charged-lepton and Majorana neutrino singlets. After spontaneous gauge symmetry breaking, we obtain the charged-lepton mass matrix $M_{l}=Y_{l} v$ and the Dirac neutrino mass matrix $M_{\mathrm{D}}=Y_{\nu} v$ with $v \approx 174 \mathrm{GeV}$ being the vacuum expectation value of $H$. Because $N_{1}$ and $N_{2}$ are $S U(2)_{\mathrm{L}}$ singlets, their mass scale is not subject to the electroweak symmetry breaking and can be much higher than $v$. Then the seesaw mechanism [6] works for the effective (light and left-handed) neutrino mass matrix $M_{\nu}$; i.e., $M_{\nu}=M_{\mathrm{D}} M_{\mathrm{R}}^{-1} M_{\mathrm{D}}^{T}$ holds as a very good approximation. The smallness of left-handed neutrino masses $m_{i}$ (for $i=1,2,3$ ) is therefore ascribed to the largeness of right-handed neutrino masses $M_{i}$ (for $i=1,2$ ), while the bi-large neutrino mixing pattern is attributed to a significant mismatch between the diagonalizations of $M_{l}$ and $M_{\nu}$.

Note that $\mathcal{L}_{\text {lepton }}$ allows the lepton-number-violating decays of $N_{i}$ (for $i=1,2$ ) to happen: $N_{i} \rightarrow l+H^{\mathrm{c}}$ and $N_{i} \rightarrow l^{\mathrm{c}}+H$. Since each decay mode can occur at both tree and one-loop levels, the interference of two decay amplitudes leads to a CP-violating asymmetry $\varepsilon_{i}$ between $N_{i} \rightarrow l+H^{\mathrm{c}}$ and its CP-conjugated process $N_{i} \rightarrow l^{\mathrm{c}}+H$. If the expansion of the Universe gives rise to the out-of-equilibrium decays of $N_{1}$ and (or) $N_{2}$ [7], then $\varepsilon_{1}$ and $\varepsilon_{2}$ (or one of them) may result in a net lepton number asymmetry. The latter can partially be converted into a net baryon number asymmetry via the nonperturbative sphaleron interaction [8]. Such an elegant baryogenesis-via-leptogenesis mechanism [9] provides a viable interpretation of the cosmological baryon number asymmetry, $\eta_{\mathrm{B}} \equiv n_{\mathrm{B}} / n_{\gamma}=(6.1 \pm 0.2) \times 10^{-10}$, which has recently been extracted from the three-year WMAP observational data [10]. 
The most straightforward test of the seesaw and leptogenesis mechanisms is to discover the heavy right-handed Majorana neutrinos and measure the strength of their interactions with other known particles, for instance, at the Large Hadron Collider (LHC) and the International Linear Collider (ILC) [11] in the near or foreseeable future. Nevertheless, $N_{i}$ cannot be produced at LHC unless its mass scale $M_{i}$ is of $\mathcal{O}(1) \mathrm{TeV}$ up to $\mathcal{O}(10) \mathrm{TeV}$, far below the typical seesaw scale $\Lambda_{\mathrm{SS}} \sim 10^{10 \cdots 14} \mathrm{GeV}[6]$. If $M_{i} \sim \mathcal{O}(1) \mathrm{TeV}$ is taken, for example, a successful leptogenesis to account for the observed value of $\eta_{\mathrm{B}}$ requires that the splitting between $M_{1}$ and $M_{2}$ be extremely small [12]. This interesting scenario, usually referred to as the resonant leptogenesis mechanism, may also get clear of the gravitino overproduction problem in those supersymmetric extensions of the standard model with heavy Majorana neutrinos of $M_{i} \sim \Lambda_{\mathrm{SS}}$. But $m_{i} \lesssim \mathcal{O}(1) \mathrm{eV}$ and $M_{i} \sim \mathcal{O}(1) \mathrm{TeV}$ in the seesaw relation imply that the corresponding Yukawa couplings must be of $\mathcal{O}\left(10^{-6}\right)$ or smaller, rendering the production and detection of $N_{i}$ rather dim at LHC and ILC [13]. For this reason, here we shall follow a less ambitious strategy by leaving out the observability of heavy right-handed Majorana neutrinos and concentrating on the phenomenology of neutrino mixing and resonant leptogenesis.

The goal of this paper is to simultaneously explain the observed neutrino mixing pattern and the cosmological baryon number asymmetry. We are going to propose a particularly economical and suggestive seesaw model with two highly degenerate right-handed Majorana neutrinos of $\mathcal{O}(1) \mathrm{TeV}$. The novel Yukawa-coupling texture of our model leads to the normal neutrino mass hierarchy with $m_{1}=0$ and a nearly tri-bimaximal neutrino mixing pattern with the maximal CP-violating phase: $\theta_{23}=\pi / 4,|\delta|=\pi / 2$ and $\sin ^{2} \theta_{12}=\left(1-2 \tan ^{2} \theta_{13}\right) / 3$. On the other hand, one may get the inverted neutrino mass hierarchy with $m_{3}=0$ and the corresponding neutrino mixing pattern with $\theta_{23}=\pi / 4$ and $\theta_{13}=\delta=0$. We find that it is possible to establish a straightforward link between CP violation in the decays of heavy right-handed Majorana neutrinos and that in the oscillations of light left-handed Majorana neutrinos in the $m_{1}=0$ case. As for the cosmological baryon number asymmetry, we show that the observed value of $\eta_{\mathrm{B}}$ can naturally be interpreted through the resonant leptogenesis mechanism in both $m_{1}=0$ and $m_{3}=0$ cases. We point out that the formula of $\varepsilon_{i}$ given in Ref. [12] and that presented in Ref. [14] lead to the same numerical result in the model under discussion, if the parameter space coincides with the perturbation condition. More interestingly, we demonstrate the significance of flavor-dependent effects in our resonant 
leptogenesis scenario: they can either flip the sign of the flavor-independent prediction for $\eta_{\mathrm{B}}$ in the $m_{1}=0$ case or magnify the magnitude of the flavor-independent prediction for $\eta_{\mathrm{B}}$ about 50 times in the $m_{3}=0$ case.

\section{NEUTRINO MIXING}

A salient feature of the minimal seesaw model is that it automatically predicts $m_{1}=0$ or $m_{3}=0$ [5]. Hence the $3 \times 2$ Dirac neutrino mass matrix $M_{\mathrm{D}}$ can be parameterized as

$$
M_{\mathrm{D}}^{(1)}=V_{0}\left(\begin{array}{ll}
0 & 0 \\
x & 0 \\
0 & y
\end{array}\right) U
$$

with $m_{1}=0$ [15] or as

$$
M_{\mathrm{D}}^{(3)}=V_{0}\left(\begin{array}{ll}
x & 0 \\
0 & y \\
0 & 0
\end{array}\right) U
$$

with $m_{3}=0$, where $V_{0}$ and $U$ are $3 \times 3$ and $2 \times 2$ unitary matrices, respectively. Without loss of generality, we adopt the flavor basis in which both the charged-lepton mass matrix $M_{l}$ and the right-handed Majorana neutrino mass matrix $M_{\mathrm{R}}$ are diagonal, real and positive. Then the seesaw relation $M_{\nu}=M_{\mathrm{D}} M_{\mathrm{R}}^{-1} M_{\mathrm{D}}^{T}$ implies that the flavor mixing of light Majorana neutrinos depends primarily on $V_{0}$ and the decays of heavy Majorana neutrinos rely mainly on $U$ [16]. This phenomenological observation motivates us to take $V_{0}$ to be the tri-bimaximal mixing pattern [17]

$$
V_{0}=\left(\begin{array}{ccc}
2 / \sqrt{6} & 1 / \sqrt{3} & 0 \\
-1 / \sqrt{6} & 1 / \sqrt{3} & 1 / \sqrt{2} \\
1 / \sqrt{6} & -1 / \sqrt{3} & 1 / \sqrt{2}
\end{array}\right),
$$

which is compatible very well with the best fit of current experimental data on neutrino oscillations [18]. On the other hand, the unitary matrix $U$ can be parameterized as

$$
U=\left(\begin{array}{cc}
\cos \vartheta & \sin \vartheta \\
-\sin \vartheta & \cos \vartheta
\end{array}\right)\left(\begin{array}{cc}
e^{-i \alpha} & 0 \\
0 & e^{+i \alpha}
\end{array}\right) .
$$

Since $\alpha$ is the only phase parameter in our model, it should be responsible both for the $\mathrm{CP}$ violation in neutrino oscillations and for the $\mathrm{CP}$ violation in $N_{i}$ decays. In order to implement the idea of resonant leptogenesis, we assume that two heavy Majorana neutrino masses are highly degenerate; i.e., the magnitude of $r \equiv\left(M_{2}-M_{1}\right) / M_{2}$ is strongly suppressed. Indeed 
$|r| \sim \mathcal{O}\left(10^{-7}\right)$ or smaller has typically been anticipated in some seesaw models with three right-handed Majorana neutrinos [12] to gain the successful resonant leptogenesis.

Given $|r|<\mathcal{O}\left(10^{-4}\right)$, the explicit form of $M_{\nu}$ can reliably be formulated from the seesaw relation $M_{\nu}=M_{\mathrm{D}} M_{\mathrm{R}}^{-1} M_{\mathrm{D}}^{T}$ by neglecting the tiny mass splitting between $N_{1}$ and $N_{2}$. In such a good approximation, we obtain

$$
M_{\nu}^{(1)}=\frac{y^{2}}{M_{2}}\left[V_{0}\left(\begin{array}{ccc}
0 & 0 & 0 \\
0 & \omega^{2}(\cos 2 \alpha-i \cos 2 \vartheta \sin 2 \alpha) & i \omega \sin 2 \vartheta \sin 2 \alpha \\
0 & i \omega \sin 2 \vartheta \sin 2 \alpha & \cos 2 \alpha+i \cos 2 \vartheta \sin 2 \alpha
\end{array}\right) V_{0}^{T}\right]
$$

for $m_{1}=0$; or

$$
\left.M_{\nu}^{(3)}=\frac{y^{2}}{M_{2}}\left[\begin{array}{ccc}
\omega^{2}(\cos 2 \alpha-i \cos 2 \vartheta \sin 2 \alpha) & i \omega \sin 2 \vartheta \sin 2 \alpha & 0 \\
i \omega \sin 2 \vartheta \sin 2 \alpha & \cos 2 \alpha+i \cos 2 \vartheta \sin 2 \alpha & 0 \\
0 & 0 & 0
\end{array}\right) V_{0}^{T}\right]
$$

for $m_{3}=0$, where $\omega \equiv x / y$ is defined. One may follow the procedure outlined in Ref. [15] to diagonalize the effective neutrino mass matrix in Eq. (5a) or (5b). For simplicity, here we fix $\vartheta=\pi / 4$ and highlight the role of $\alpha$ in neutrino mixing and leptogenesis. Then both the neutrino mass spectrum and the neutrino mixing pattern can be achieved from the simplified version of $M_{\nu}^{(1)}$ or $M_{\nu}^{(3)}$.

- Normal neutrino mass hierarchy with $m_{1}=0$. In this case, we diagonalize $M_{\nu}^{(1)}$ by using the transformation $V^{\dagger} M_{\nu}^{(1)} V^{*}=\operatorname{Diag}\left\{0, m_{2}, m_{3}\right\}$, where $V$ is just the neutrino mixing matrix. After a straightforward calculation, we have

$$
\begin{aligned}
& m_{2}=\frac{y^{2}}{2 M_{2}}\left[\sqrt{\left(1+\omega^{2}\right)^{2} \cos ^{2} 2 \alpha+4 \omega^{2} \sin ^{2} 2 \alpha}-\left(1-\omega^{2}\right)|\cos 2 \alpha|\right], \\
& m_{3}=\frac{y^{2}}{2 M_{2}}\left[\sqrt{\left(1+\omega^{2}\right)^{2} \cos ^{2} 2 \alpha+4 \omega^{2} \sin ^{2} 2 \alpha}+\left(1-\omega^{2}\right)|\cos 2 \alpha|\right],
\end{aligned}
$$

where $0<\omega<1$. Taking account of $m_{2}=\sqrt{\Delta m_{21}^{2}}$ and $m_{3}=\sqrt{\Delta m_{21}^{2}+\left|\Delta m_{32}^{2}\right|}$, we obtain $m_{2} \approx 8.9 \times 10^{-3} \mathrm{eV}$ and $m_{3} \approx 5.1 \times 10^{-2} \mathrm{eV}$ by using $\Delta m_{21}^{2} \approx 8.0 \times 10^{-5} \mathrm{eV}^{2}$ and $\left|\Delta m_{32}^{2}\right| \approx 2.5 \times 10^{-3} \mathrm{eV}^{2}[18]$ as the typical inputs. Furthermore,

$$
V=\left(\begin{array}{ccc}
2 / \sqrt{6} & \cos \theta / \sqrt{3} & i \sin \theta / \sqrt{3} \\
-1 / \sqrt{6} & \cos \theta / \sqrt{3}+i \sin \theta / \sqrt{2} & \cos \theta / \sqrt{2}+i \sin \theta / \sqrt{3} \\
1 / \sqrt{6} & -\cos \theta / \sqrt{3}+i \sin \theta / \sqrt{2} & \cos \theta / \sqrt{2}-i \sin \theta / \sqrt{3}
\end{array}\right),
$$

where $\theta$ is given by $\tan 2 \theta=2 \omega \tan 2 \alpha /\left(1+\omega^{2}\right)$. Comparing this result with the standard parametrization of $V$ [19], we immediately arrive at

$$
\sin ^{2} \theta_{12}=\frac{1-\sin ^{2} \theta}{3-\sin ^{2} \theta}, \quad \sin ^{2} \theta_{13}=\frac{\sin ^{2} \theta}{3}, \quad \theta_{23}=\frac{\pi}{4}, \quad \delta=-\frac{\pi}{2},
$$


and vanishing Majorana phases of $\mathrm{CP}$ violation. Eq. (8a) implies a very interesting correlation between $\theta_{12}$ and $\theta_{13}: \sin ^{2} \theta_{12}=\left(1-2 \tan ^{2} \theta_{13}\right) / 3$. When $\theta_{13} \rightarrow 10^{\circ}$, we get $\theta_{12} \rightarrow 34^{\circ}$, which is almost the best-fit value of the solar neutrino mixing angle [18] (i.e., the large-mixing-angle MSW solution to the solar neutrino problem [20]). Note that the smallness of $\theta_{13}$ requires the smallness of $\theta$, or equivalently the smallness of $\alpha$. Eqs. (6a) and (8a), together with $\theta_{13}<10^{\circ}$ and the values of $m_{2}$ and $m_{3}$ obtained above, yield $0.39 \lesssim \omega \lesssim 0.42,0^{\circ} \lesssim \alpha \lesssim 23^{\circ}$ and $0^{\circ} \lesssim \theta \lesssim 18^{\circ}$. Eq. (6a) can reliably approximate to $m_{2} \approx x^{2} / M_{2}$ and $m_{3} \approx y^{2} / M_{2}$ for $\alpha \lesssim 10^{\circ}$. The Jarlskog rephasinginvariant parameter $\mathcal{J}$ [21], which determines the strength of $\mathrm{CP}$ violation in neutrino oscillations, is found to be $|\mathcal{J}|=\sin 2 \theta /(6 \sqrt{6}) \lesssim 0.04$ in this scenario. It is possible to measure $|\mathcal{J}| \sim \mathcal{O}\left(10^{-2}\right)$ in the future long-baseline neutrino oscillation experiments.

- Inverted neutrino mass hierarchy with $m_{3}=0$. In this case, we diagonalize $M_{\nu}^{(3)}$ by using the transformation $V^{\dagger} M_{\nu}^{(3)} V^{*}=\operatorname{Diag}\left\{m_{1}, m_{2}, 0\right\}$. Then we obtain

$$
\begin{aligned}
& m_{1}=\frac{y^{2}}{2 M_{2}}\left[\sqrt{\left(1+\omega^{2}\right)^{2} \cos ^{2} 2 \alpha+4 \omega^{2} \sin ^{2} 2 \alpha}-\left(1-\omega^{2}\right)|\cos 2 \alpha|\right], \\
& m_{2}=\frac{y^{2}}{2 M_{2}}\left[\sqrt{\left(1+\omega^{2}\right)^{2} \cos ^{2} 2 \alpha+4 \omega^{2} \sin ^{2} 2 \alpha}+\left(1-\omega^{2}\right)|\cos 2 \alpha|\right],
\end{aligned}
$$

where $0<\omega<1$. Taking account of $m_{1}=\sqrt{\left|\Delta m_{32}^{2}\right|-\Delta m_{21}^{2}}$ and $m_{2}=\sqrt{\left|\Delta m_{32}^{2}\right|}$, we get $m_{1} \approx 4.9 \times 10^{-2} \mathrm{eV}$ and $m_{2} \approx 5.0 \times 10^{-2} \mathrm{eV}$ by inputting $\Delta m_{21}^{2} \approx 8.0 \times 10^{-5} \mathrm{eV}^{2}$ and $\left|\Delta m_{32}^{2}\right| \approx 2.5 \times 10^{-3} \mathrm{eV}^{2}$. In addition,

$$
V=\left(\begin{array}{ccc}
2 \cos \theta / \sqrt{6}+i \sin \theta / \sqrt{3} & \cos \theta / \sqrt{3}+2 i \sin \theta / \sqrt{6} & 0 \\
-\cos \theta / \sqrt{6}+i \sin \theta / \sqrt{3} & \cos \theta / \sqrt{3}-i \sin \theta / \sqrt{6} & 1 / \sqrt{2} \\
\cos \theta / \sqrt{6}-i \sin \theta / \sqrt{3} & -\cos \theta / \sqrt{3}+i \sin \theta / \sqrt{6} & 1 / \sqrt{2}
\end{array}\right)
$$

where $\theta$ is given by $\tan 2 \theta=2 \omega \tan 2 \alpha /\left(1+\omega^{2}\right)$. When the standard parametrization of $V$ [19] is applied to Eq. (7b), we have

$$
\sin ^{2} \theta_{12}=\frac{1+\sin ^{2} \theta}{3}, \quad \theta_{13}=0, \quad \theta_{23}=\frac{\pi}{4}, \quad \delta=0, \quad \mathcal{J}=0,
$$

and vanishing Majorana phases of CP violation. Eq. (8b) indicates that $M_{\nu}^{(3)}$ actually has the $\mu-\tau$ symmetry and $\mathrm{CP}$ is conserving at low-energy scales in this scenario. Given $30^{\circ}<\theta_{12}<38^{\circ}$ [18], $\theta$ is found to lie in the range $0 \lesssim \theta \lesssim 22^{\circ}$. This result, together with Eq. (6b) and the values of $m_{1}$ and $m_{2}$ obtained above, allows us to get $0^{\circ} \lesssim \alpha \lesssim 22^{\circ}$ and $0.991 \lesssim \omega \lesssim 0.992$. We observe that $m_{1} \approx x^{2} / M_{2}$ and $m_{2} \approx y^{2} / M_{2}$ are good approximations of Eq. (6b) for $\alpha \lesssim 10^{\circ}$. 
From a phenomenological point of view, the scenario with $m_{1}=0$ is more favored and more interesting than the scenario with $m_{3}=0$. Both of them can be tested in the near future.

\section{RESONANT LEPTOGENESIS}

Now let us consider the lepton-number-violating and CP-violating decays of two heavy right-handed Majorana neutrinos. Their decay widths are given by $\Gamma_{i}=\left(Y_{\nu}^{\dagger} Y_{\nu}\right)_{i i} M_{i} /(8 \pi)$ at the tree level, where $Y_{\nu}=M_{\mathrm{D}} / v$. With the help of Eq. (2), it is easy to verify that $M_{\mathrm{D}}^{\dagger} M_{\mathrm{D}}$ has a universal form for both $m_{1}=0$ and $m_{3}=0$ cases:

$$
M_{\mathrm{D}}^{\dagger} M_{\mathrm{D}}=U^{\dagger}\left(\begin{array}{cc}
x^{2} & 0 \\
0 & y^{2}
\end{array}\right) U
$$

Given the near degeneracy between $M_{1}$ and $M_{2}$ as well as $\vartheta=\pi / 4$ for $U$, it turns out that $\Gamma_{1}=\Gamma_{2}$ is an excellent approximation. But the decay mode $N_{i} \rightarrow l+H^{\mathrm{c}}$ and its CPconjugated process $N_{i} \rightarrow l^{\mathrm{c}}+H$ can actually occur at both tree and one-loop levels. Hence their CP-violating asymmetry $\varepsilon_{i}$, defined as the ratio of $\Gamma\left(N_{i} \rightarrow l+H^{\mathrm{c}}\right)-\Gamma\left(N_{i} \rightarrow l^{\mathrm{c}}+H\right)$ to $\Gamma\left(N_{i} \rightarrow l+H^{\mathrm{c}}\right)+\Gamma\left(N_{i} \rightarrow l^{\mathrm{c}}+H\right)$, arises from the interference of two decay amplitudes [9]. Note that the self-energy correction dominates over the vertex correction at the one-loop level, as the former is resonantly enhanced by the tiny mass splitting between $N_{1}$ and $N_{2}$. In other words, $\varepsilon_{i}$ primarily results from the interference between the tree-level amplitude and its self-energy correction [12] [31],

$$
\varepsilon_{i}=\frac{\operatorname{Im}\left[\left(Y_{\nu}^{\dagger} Y_{\nu}\right)_{i j}^{2}\right]}{\left(Y_{\nu}^{\dagger} Y_{\nu}\right)_{11}\left(Y_{\nu}^{\dagger} Y_{\nu}\right)_{22}} \cdot \frac{\left(M_{i}^{2}-M_{j}^{2}\right) M_{i} \Gamma_{j}}{\left(M_{i}^{2}-M_{j}^{2}\right)^{2}+M_{i}^{2} \Gamma_{j}^{2}}
$$

where $i$ and $j$ run over 1 and 2 but $i \neq j$. Combining Eqs. (9) and (10), we obtain the explicit expression of $\varepsilon_{i}$ in our model:

$$
\varepsilon_{i}=\frac{-32 \pi v^{2} y^{2}\left(1-\omega^{2}\right)^{2}}{\left(1+\omega^{2}\right)\left[1024 \pi^{2} v^{4} r^{2}+y^{4}\left(1+\omega^{2}\right)^{2}\right]} r \sin 4 \alpha,
$$

in which $r \equiv\left(M_{2}-M_{1}\right) / M_{2}$ has been defined to describe the mass splitting between two heavy Majorana neutrinos. Of course, $\varepsilon_{1}=\varepsilon_{2}$ is also an excellent approximation due to $\vartheta=45^{\circ}$. These two CP-violating asymmetries would vanish if $r=0$ or $\alpha=0$ were taken.

To estimate the order of $\varepsilon_{i}$ at the TeV scale, we restrict ourselves to the interesting $\alpha \lesssim 10^{\circ}$

region and make use of the approximate result $y^{2} \approx m_{3} M_{2}$ (or $y^{2} \approx m_{2} M_{2}$ ) obtained above for the $m_{1}=0$ (or $\left.m_{3}=0\right)$ case. 
- In the $m_{1}=0$ case, we get $y^{2} \approx 5.1 \times 10^{-8} \mathrm{GeV}^{2}$ from $m_{3} \approx 5.1 \times 10^{-2} \mathrm{eV}$ and $M_{2} \approx 1$ $\mathrm{TeV}$. In addition, $\omega \approx 0.42$. Then Eq. (11) is approximately simplified to

$$
\varepsilon_{i} \approx \begin{cases}-9.7 \times 10^{-15} r^{-1} \sin 4 \alpha, & \text { for } r \gg 2.0 \times 10^{-14}, \\ -2.5 \times 10^{13} r \sin 4 \alpha, & \text { for } r \ll 2.0 \times 10^{-14},\end{cases}
$$

together with $\varepsilon_{i} \sim-0.25 \times \sin 4 \alpha$ for $r \sim 2.0 \times 10^{-14}$. Note that $\left|\varepsilon_{i}\right| \sim \mathcal{O}\left(10^{-5}\right)$ is in general expected so as to achieve the successful leptogenesis (see below). Hence the third possibility $r \sim \mathcal{O}\left(10^{-14}\right)$ requires $\alpha \sim \mathcal{O}\left(10^{-4}\right)$, implying very tiny (unobservable) $\mathrm{CP}$ violation in neutrino oscillations. If $\alpha \sim 5^{\circ} \cdots 10^{\circ}$, one may take either $r \sim 10^{-10}$ or $r \sim 10^{-18}$ to obtain $\left|\varepsilon_{i}\right| \sim \mathcal{O}\left(10^{-5}\right)$.

- In the $m_{3}=0$ case, we obtain $y^{2} \approx 5.0 \times 10^{-8} \mathrm{GeV}^{2}$ from $m_{2} \approx 5.0 \times 10^{-2} \mathrm{eV}$ and $M_{2} \approx 1 \mathrm{TeV}$. In addition, $\omega \approx 0.99$. Then Eq. (11) is approximately simplified to

$$
\varepsilon_{i} \approx \begin{cases}-3.3 \times 10^{-18} r^{-1} \sin 4 \alpha, & \text { for } r \gg 3.2 \times 10^{-14}, \\ -3.1 \times 10^{9} r \sin 4 \alpha, & \text { for } r \ll 3.2 \times 10^{-14},\end{cases}
$$

together with $\varepsilon_{i} \sim-5.0 \times 10^{-5} \sin 4 \alpha$ for $r \sim 3.2 \times 10^{-14}$. Given either $r \sim \mathcal{O}\left(10^{-14}\right)$ or $r \sim \mathcal{O}\left(10^{-13}\right)$, it is straightforward to arrive at $\left|\varepsilon_{i}\right| \sim \mathcal{O}\left(10^{-5}\right)$ for $\alpha \sim 5^{\circ} \cdots 10^{\circ}$. If $r \ll 3.2 \times 10^{-14}$ holds, on the other hand, $\left|\varepsilon_{i}\right|$ will be impossible to reach $\mathcal{O}\left(10^{-5}\right)$. Note again that $\mathcal{J}=0$ has been obtained in Eq. (8b), implying that $\varepsilon_{i} \neq 0$ is completely independent of $\mathcal{J}=0$ in this scenario.

As described before, the CP-violating asymmetries $\varepsilon_{1}$ and $\varepsilon_{2}$ can give rise to a net lepton number asymmetry in the Universe, provided its expansion rate is larger than $\Gamma_{1}$ and $\Gamma_{2}$. The nonperturbative sphaleron interaction may partially convert this lepton number asymmetry into a net baryon number asymmetry [22],

$$
\eta_{\mathrm{B}} \approx-0.96 \times 10^{-2} \sum_{i}\left(\kappa_{i} \varepsilon_{i}\right) \approx-1.92 \times 10^{-2} \kappa_{i} \varepsilon_{i},
$$

where $\kappa_{1}$ and $\kappa_{2}$ are the efficiency factors measuring the washout effects associated with the out-of-equilibrium decays of $N_{1}$ and $N_{2}$, and $\kappa_{1}=\kappa_{2}$ is an excellent approximation in our model. To evaluate the magnitude of $\kappa_{i}$, let us take account of the effective neutrino masses $\tilde{m}_{i} \equiv\left(M_{\mathrm{D}}^{\dagger} M_{\mathrm{D}}\right)_{i i} / M_{i}=\left(x^{2}+y^{2}\right) /\left(2 M_{i}\right)$. Of course, $\tilde{m}_{1}=\tilde{m}_{2}$ is also an excellent approximation. Given $\alpha \lesssim 10^{\circ}$,

$$
\tilde{m}_{i} \approx \frac{1}{2}\left(m_{2}+m_{3}\right) \approx 2.9 \times 10^{-2} \mathrm{eV}
$$


holds for the normal neutrino mass hierarchy $\left(m_{1}=0\right)$; and

$$
\tilde{m}_{i} \approx \frac{1}{2}\left(m_{1}+m_{2}\right) \approx 5.0 \times 10^{-2} \mathrm{eV}
$$

holds for the inverted neutrino mass hierarchy $\left(m_{3}=0\right)$. When $\tilde{m}_{i}>10^{-3} \mathrm{eV}$, the washout of the baryon number asymmetry is so effective that the final asymmetry can hardly depend on the initial one. Alternatively, one may define the parameters $K_{i} \equiv \Gamma_{i} / H$ at $T=M_{i}$, where $T$ denotes the temperature of the Universe, $H \equiv 1.66 \sqrt{g_{*}} T^{2} / M_{\text {Planck }}$ is the Hubble constant with $g_{*} \simeq 107$, and $M_{\text {Planck }} \approx 1.2 \times 10^{19} \mathrm{GeV}$ represents the Planck mass. The relationship between $K_{i}$ and $\tilde{m}_{i}$ is rather simple: $K_{i}=\tilde{m}_{i} / m_{*}$, where $m_{*} \simeq 1.08 \times 10^{-3} \mathrm{eV}$ is the so-called equilibrium neutrino mass. For simplicity, the efficiency factors $\kappa_{i}$ can be estimated by using the approximate formula [23]

$$
\kappa_{1} \approx \kappa_{2} \approx \frac{1}{2}\left(\sum_{i} K_{i}\right)^{-1.2}
$$

which is valid when the masses of two heavy right-handed Majorana neutrinos are nearly degenerate and the parameters $K_{i}$ lie in the range $5 \lesssim K_{i} \lesssim 100$. With the help of Eq. (14), we get $K_{i} \approx 27$ and $\kappa_{i} \approx 4.2 \times 10^{-3}$ for the $m_{1}=0$ case; or $K_{i} \approx 46$ and $\kappa_{i} \approx 2.2 \times 10^{-3}$ for the $m_{3}=0$ case. Finally, the cosmological baryon number asymmetry is found to be

$$
\eta_{\mathrm{B}} \approx \begin{cases}7.8 \times 10^{-19} r^{-1} \sin 4 \alpha, & \text { for } r \gg 2.0 \times 10^{-14} \\ 2.0 \times 10^{9} r \sin 4 \alpha, & \text { for } r \ll 2.0 \times 10^{-14}\end{cases}
$$

in the $m_{1}=0$ case; or

$$
\eta_{\mathrm{B}} \approx \begin{cases}1.4 \times 10^{-22} r^{-1} \sin 4 \alpha, & \text { for } r \gg 3.2 \times 10^{-14} \\ 1.3 \times 10^{5} r \sin 4 \alpha, & \text { for } r \ll 3.2 \times 10^{-14}\end{cases}
$$

in the $m_{3}=0$ case.

Note that the numerical results in Eq. (16) are obtained by taking $M_{2} \approx 1 \mathrm{TeV}$. Other numerical results can similarly be achieved by starting from Eq. (11) and allowing $M_{2}$ to vary, for instance, from $1 \mathrm{TeV}$ to $10 \mathrm{TeV}$. It is certainly possible to get $\eta_{\mathrm{B}} \approx 6.1 \times 10^{-10}$ from Eq. (16) with a proper choice of $r$ and $\alpha$, either in the $m_{1}=0$ case or in the $m_{3}=0$ case. But one should keep in mind that the flavor-independent leptogenesis scenario under discussion has a potential problem, because it ignores the fact that all the Yukawa interactions of charged leptons are in thermal equilibrium at temperatures of $\mathcal{O}(1) \mathrm{TeV}$ [24]. We shall 
consider the flavor-dependent effect in the next section and demonstrate its significance in our resonant leptogenesis model.

We remark that the formula of $\varepsilon_{i}$ in Eq. (10) is quoted from Ref. [12]. A different formula has recently been presented in Ref. [14] to describe the resonant CP-violating asymmetry for two nearly degenerate right-handed Majorana neutrinos:

$$
\varepsilon_{i}^{\prime}=\frac{\operatorname{Im}\left[\left(Y_{\nu}^{\dagger} Y_{\nu}\right)_{i j}^{2}\right]}{\left(Y_{\nu}^{\dagger} Y_{\nu}\right)_{11}\left(Y_{\nu}^{\dagger} Y_{\nu}\right)_{22}} \cdot \frac{\left(M_{i}^{2}-M_{j}^{2}\right) M_{i} \Gamma_{j}}{\left(M_{i}^{2}-M_{j}^{2}\right)^{2}+\left(M_{j} \Gamma_{j}-M_{i} \Gamma_{i}\right)^{2}}
$$

where the perturbation condition $\left(M_{2}-M_{1}\right) / M_{2} \gg \max \left[\left(Y_{\nu}^{\dagger} Y_{\nu}\right)_{i j} /\left(16 \pi^{2}\right)\right]$ should be satisfied. Which formula is more reliable remains controversial at present. Here let us compare between the results of $\varepsilon_{i}$ and $\varepsilon_{i}^{\prime}$ in our model. Taking account of

$$
M_{j} \Gamma_{j}-M_{i} \Gamma_{i}=\hat{r}\left(M_{j}^{2}-M_{i}^{2}\right)
$$

where $\hat{r} \equiv \Gamma_{i} / M_{i}=\Gamma_{j} / M_{j}=y^{2}\left(1+\omega^{2}\right) /\left(16 \pi v^{2}\right)$ for $i \neq j$, we obtain the ratio

$$
\frac{\varepsilon_{i}^{\prime}}{\varepsilon_{i}}=\frac{\left(M_{i}^{2}-M_{j}^{2}\right)^{2}+M_{i}^{2} \Gamma_{j}^{2}}{\left(M_{i}^{2}-M_{j}^{2}\right)^{2}+\left(M_{j} \Gamma_{j}-M_{i} \Gamma_{i}\right)^{2}}=\frac{1+\hat{r}^{2} /\left(4 r^{2}\right)}{1+\hat{r}^{2}}
$$

from Eqs. (10) and (17) as an excellent approximation. Note that $\hat{r} / 2 \approx 2.0 \times 10^{-14}$ (for $m_{1}=0$ ) or $\hat{r} / 2 \approx 3.2 \times 10^{-14}$ (for $m_{3}=0$ ) in the numerical examples taken above. Note also that the perturbation condition used in deriving Eq. (17) is equivalent to $r \gg \hat{r} /(2 \pi)$. Hence we arrive at $\varepsilon_{i}^{\prime} \approx \varepsilon_{i}$ when $r \gg \hat{r} / 2$ holds, or equivalently $r \gg \hat{r} /(2 \pi)$ is satisfied. In other words, the formula of $\varepsilon_{i}$ given in Ref. [12] and that presented in Ref. [14] lead to the same numerical results in our leptogenesis scenario, if and only if the parameter space coincides with the perturbation condition. This numerical agreement is by no means a hint that there might not exist any serious discrepancy between Refs. [12] and [14]. It is actually desirable to understand the properties of unstable particles in quantum field theories to the utmost extent, so as to clarify the potential ambiguities associated with $\varepsilon_{i}$ and $\varepsilon_{i}^{\prime}$ (or one of them) in the resonant leptogenesis models.

\section{FLAVOR EFFECTS}

Flavor-dependent effects in leptogenesis have recently attracted a lot of attention [25]. Since all the Yukawa interactions of charged leptons are in thermal equilibrium at the TeV 
scale, the flavor issues of $N_{i}$ decays should be taken into account in our model. In the framework of resonant leptogenesis, it is straightforward to calculate the CP-violating asymmetry between $N_{i} \rightarrow l_{\alpha}+H^{\mathrm{c}}$ and $N_{i} \rightarrow l_{\alpha}^{\mathrm{c}}+H$ decays for each lepton flavor $\alpha(=e, \mu$ or $\tau)$ :

$$
\begin{aligned}
\varepsilon_{i \alpha} & \equiv \frac{\Gamma\left(N_{i} \rightarrow l_{\alpha}+H^{\mathrm{c}}\right)-\Gamma\left(N_{i} \rightarrow l_{\alpha}^{\mathrm{c}}+H\right)}{\sum_{\alpha}\left[\Gamma\left(N_{i} \rightarrow l_{\alpha}+H^{\mathrm{c}}\right)+\Gamma\left(N_{i} \rightarrow l_{\alpha}^{\mathrm{c}}+H\right)\right]} \\
& =\frac{8 \pi\left(M_{i}^{2}-M_{j}^{2}\right) \operatorname{Im}\left\{\left(Y_{\nu}\right)_{\alpha j}\left(Y_{\nu}\right)_{\alpha i}^{*} M_{i}\left[M_{i}\left(Y_{\nu}^{\dagger} Y_{\nu}\right)_{j i}+M_{j}\left(Y_{\nu}^{\dagger} Y_{\nu}\right)_{i j}\right]\right\}}{\left[64 \pi^{2}\left(M_{i}^{2}-M_{j}^{2}\right)^{2}+M_{i}^{4}\left(Y_{\nu}^{\dagger} Y_{\nu}\right)_{j j}^{2}\right]\left(Y_{\nu}^{\dagger} Y_{\nu}\right)_{i i}},
\end{aligned}
$$

where $i$ and $j$ run over 1 and 2 but $i \neq j$. Combining Eqs. (2) and (20), we explicitly obtain

$$
\varepsilon_{i e}=\frac{\omega^{2}}{3\left(\omega^{2}-1\right)} \varepsilon_{i}, \quad \varepsilon_{i \mu}=\varepsilon_{i \tau}=\frac{2 \omega^{2}-3}{6\left(\omega^{2}-1\right)} \varepsilon_{i}
$$

in the $m_{1}=0$ case; and

$$
\varepsilon_{i e}=\frac{2 \omega^{2}-1}{3\left(\omega^{2}-1\right)} \varepsilon_{i}, \quad \varepsilon_{i \mu}=\varepsilon_{i \tau}=\frac{\omega^{2}-2}{6\left(\omega^{2}-1\right)} \varepsilon_{i}
$$

in the $m_{3}=0$ case, where $\varepsilon_{i}$ has been given in Eq. (11). Because $\varepsilon_{1}=\varepsilon_{2}$ is an excellent approximation, one can see from Eq. (21) that $\varepsilon_{1 \alpha}=\varepsilon_{2 \alpha}$ is also an excellent approximation. In view of $0.39 \lesssim \omega \lesssim 0.42$ for $m_{1}=0$ or $0.991 \lesssim \omega \lesssim 0.992$ for $m_{3}=0$, we have $\varepsilon_{i e}<0$ and $\varepsilon_{i \mu}=\varepsilon_{i \tau}>0$ in both cases. The sum of these three flavor-dependent asymmetries is just the total CP-violating asymmetry $\varepsilon_{i}$; i.e., $\varepsilon_{i e}+\varepsilon_{i \mu}+\varepsilon_{i \tau}=\varepsilon_{i}$ holds exactly.

Once the initial values of $\varepsilon_{i \alpha}$ are fixed, the final result of $\eta_{\mathrm{B}}$ will be governed by a set of flavor-dependent Boltzmann equations including the (inverse) decay and scattering processes as well as the nonperturbative sphaleron interaction [13, 24, 25]. In order to estimate the washout effects, one may introduce the parameters

$$
K_{i \alpha}=\frac{\Gamma\left(N_{i} \rightarrow l_{\alpha}+H^{\mathrm{c}}\right)+\Gamma\left(N_{i} \rightarrow l_{\alpha}^{\mathrm{c}}+H\right)}{\sum_{\alpha}\left[\Gamma\left(N_{i} \rightarrow l_{\alpha}+H^{\mathrm{c}}\right)+\Gamma\left(N_{i} \rightarrow l_{\alpha}^{\mathrm{c}}+H\right)\right]} K_{i}=\frac{\left|\left(Y_{\nu}\right)_{\alpha i}\right|^{2}}{\left(Y_{\nu}^{\dagger} Y_{\nu}\right)_{i i}} K_{i},
$$

in which $K_{i} \equiv \Gamma_{i} / H$ at $T=M_{i}$ has been defined before. Of course, $K_{i e}+K_{i \mu}+K_{i \tau}=K_{i}$ holds. With the help of Eq. (2), we get either

$$
\begin{aligned}
& K_{1 e}=K_{2 e}=\frac{\omega^{2}}{3\left(1+\omega^{2}\right)} K_{i} \\
& K_{1 \mu}=K_{2 \tau}=\frac{2 \omega^{2}-2 \sqrt{6} \omega+3}{6\left(1+\omega^{2}\right)} K_{i}, \\
& K_{1 \tau}=K_{2 \mu}=\frac{2 \omega^{2}+2 \sqrt{6} \omega+3}{6\left(1+\omega^{2}\right)} K_{i},
\end{aligned}
$$


in the $m_{1}=0$ case; or

$$
\begin{aligned}
& K_{1 e}=\frac{1-2 \sqrt{2} \omega+2 \omega^{2}}{3\left(1+\omega^{2}\right)} K_{i}, \\
& K_{1 \mu}=K_{1 \tau}=\frac{2+2 \sqrt{2} \omega+\omega^{2}}{6\left(1+\omega^{2}\right)} K_{i}, \\
& K_{2 e}=\frac{1+2 \sqrt{2} \omega+2 \omega^{2}}{3\left(1+\omega^{2}\right)} K_{i}, \\
& K_{2 \mu}=K_{2 \tau}=\frac{2-2 \sqrt{2} \omega+\omega^{2}}{6\left(1+\omega^{2}\right)} K_{i},
\end{aligned}
$$

in the $m_{3}=0$ case. The flavor-dependent effects in leptogenesis with almost degenerate heavy Majorana neutrinos have been elucidated in Ref. [23], where a useful approximation for the efficiency factor of each flavor is given as

$$
\kappa_{i \alpha} \approx \frac{2}{K_{\alpha} z_{\mathrm{B}}\left(K_{\alpha}\right)}\left[1-\exp \left(-\frac{K_{\alpha} z_{\mathrm{B}}\left(K_{\alpha}\right)}{2}\right)\right]
$$

with $z_{\mathrm{B}}\left(K_{\alpha}\right) \simeq 2+4 K_{\alpha}^{0.13} \exp \left(-2.5 / K_{\alpha}\right)$ and $K_{\alpha}=K_{1 \alpha}+K_{2 \alpha}$. The above expression can well approximate to the power law $\kappa_{i \alpha} \simeq 0.5 / K_{\alpha}^{1.2}$ for $5 \lesssim K_{\alpha} \lesssim 100$. Recalling $0.39 \lesssim \omega \lesssim 0.42$ (or $0.991 \lesssim \omega \lesssim 0.992$ ) and $K_{i} \approx 27$ (or $K_{i} \approx 46$ ) for $m_{1}=0$ (or $m_{3}=0$ ), one can then evaluate the decay parameters $K_{\alpha}: K_{e} \approx 2.4$ and $K_{\mu}=K_{\tau} \approx 25.8$ (or $K_{e} \approx 46$ and $\left.K_{\mu}=K_{\tau} \approx 23\right)$. The final baryon number asymmetry can be calculated via

$$
\eta_{\mathrm{B}}^{\mathrm{f}} \approx-0.96 \times 10^{-2} \sum_{i} \sum_{\alpha}\left(\varepsilon_{i \alpha} \kappa_{i \alpha}\right),
$$

where the superscript means that flavor effects have been included; $\varepsilon_{i \alpha}$ and $\kappa_{i \alpha}$ are given in Eqs. (21) and (24), respectively. A straightforward comparison between $\eta_{\mathrm{B}}$ in Eq. (13) and $\eta_{\mathrm{B}}^{\mathrm{f}}$ in Eq. (25) can clearly demonstrate the significance of flavor effects in our resonant leptogenesis scenario:

$$
\frac{\eta_{\mathrm{B}}^{\mathrm{f}}}{\eta_{\mathrm{B}}}=\frac{\sum_{i} \sum_{\alpha}\left(\varepsilon_{i \alpha} \kappa_{i \alpha}\right)}{\sum_{i}\left(\varepsilon_{i} \kappa_{i}\right)} \approx\left\{\begin{array}{cc}
-0.71 & \left(m_{1}=0\right), \\
52.1 & \left(m_{3}=0\right) .
\end{array}\right.
$$

Note that flavor effects flip the sign of $\eta_{\mathrm{B}}$ in the $m_{1}=0$ case. This nontrivial result can be understood as follows. The novel Yukawa structure of our model dictates the electron flavor to make a negative contribution to the CP-violating asymmetry $\left(\varepsilon_{i e}<0\right)$ and to have a large efficiency factor (due to the smallness of $K_{e}$; i.e., $K_{e} \approx 2.4$ ). Although the other two flavors contribute significantly to the $\mathrm{CP}$-violating asymmetry, their corresponding washout effects are much stronger $\left(K_{\mu}=K_{\tau} \approx 25.8\right)$. Hence the overall effects of three flavors in $\eta_{\mathrm{B}}^{\mathrm{f}}$ 
make its sign opposite to that of $\eta_{\mathrm{B}}$ in the $m_{1}=0$ case. In the $m_{3}=0$ case, the large and negative contribution of the electron flavor to the CP-violating asymmetry is badly diluted by its extremely small efficiency factor (due to the largeness of $K_{e}$; i.e., $K_{e} \approx 46$ ), and thus the muon and tau flavors make the dominant contributions to $\eta_{\mathrm{B}}^{\mathrm{f}}$ via both $\varepsilon_{i \mu}=\varepsilon_{i \tau}$ and $\kappa_{i \mu}=\kappa_{i \tau}$. That is why $\eta_{\mathrm{B}}^{\mathrm{f}}$ has the same sign as $\eta_{\mathrm{B}}$ in the $m_{3}=0$ case, in which the large ratio $\eta_{\mathrm{B}}^{\mathrm{f}} / \eta_{\mathrm{B}} \approx 52.1$ is essentially ascribed to the large enhancement factors $\varepsilon_{i \mu} / \varepsilon_{i} \sim 10$ and $\left(K_{1}+K_{2}\right) / K_{\mu} \sim 4$

Combining Eqs. (16) and (26), we are then able to obtain the observed value of $\eta_{\mathrm{B}}^{\mathrm{f}}$ via the flavor-dependent $\mathrm{TeV}$-scale leptogenesis with proper inputs of $r$ and $\alpha$. As an example, FIG. 1 illustrates the numerical correlation between $r$ and $\alpha$ to get $\eta_{\mathrm{B}}^{\mathrm{f}}=6.1 \times 10^{-10}$ in both $m_{1}=0$ and $m_{3}=0$ cases, where $M_{2}=1 \mathrm{TeV}, 2 \mathrm{TeV}, 3 \mathrm{TeV}, 4 \mathrm{TeV}$ and $5 \mathrm{TeV}$ have typically been input and Eq. (11) has also been used. Note that we only concentrate

on the region $r \gg \mathcal{O}\left(10^{-14}\right)$, which satisfies the perturbation condition given in Ref. [14]. One may clearly see the similar behavior of $r$ changing with $\alpha$ in both cases, in which $\eta_{\mathrm{B}}^{\mathrm{f}} \propto \varepsilon_{i} \propto y^{2} r^{-1} \sin 4 \alpha \propto M_{2} r^{-1} \sin 4 \alpha$ holds as the leading-order approximation. In order to get the correct sign for the cosmological baryon number asymmetry, however, we should take $-10^{\circ}<\alpha<0^{\circ}$ in the $m_{1}=0$ case. Since negative $\alpha$ leads to negative $\theta$ in Eq. (7a), the sign of $\delta$ in Eq. (8a) will flip. But this modification does not change the results of neutrino masses and flavor mixing angles obtained in Eqs. (6a) and (8a).

\section{FURTHER DISCUSSIONS}

In summary, we have proposed a very simple but suggestive seesaw model with two highly degenerate right-handed Majorana neutrinos of $\mathcal{O}(1) \mathrm{TeV}$. Its novel Yukawa-coupling texture leads to the normal neutrino mass hierarchy with $m_{1}=0$ and a nearly tri-bimaximal neutrino mixing pattern with the maximal CP-violating phase: $\theta_{23}=\pi / 4,|\delta|=\pi / 2$ and $\sin ^{2} \theta_{12}=\left(1-2 \tan ^{2} \theta_{13}\right) / 3$. On the other hand, it is possible to get the inverted neutrino mass hierarchy with $m_{3}=0$ and the corresponding neutrino mixing pattern with $\theta_{23}=\pi / 4$ and $\theta_{13}=\delta=0$. A straightforward link between $\mathrm{CP}$ violation in the decays of heavy right-handed Majorana neutrinos and that in the oscillations of light left-handed Majorana neutrinos can be established in the $m_{1}=0$ case. We have successfully interpreted the cosmological matter-antimatter asymmetry $\eta_{\mathrm{B}} \approx 6.1 \times 10^{-10}$ through the flavor-dependent 
resonant leptogenesis mechanism in both $m_{1}=0$ and $m_{3}=0$ cases. In particular, we have shown that flavor effects can either flip the sign of the flavor-independent prediction for $\eta_{\mathrm{B}}$ in the $m_{1}=0$ case or magnify the magnitude of the flavor-independent prediction for $\eta_{\mathrm{B}}$ about 50 times in the $m_{3}=0$ case.

Some more discussions, remarks and comments on our model are in order.

- We have made three assumptions in building the model. Taking $V_{0}$ to be the tribimaximal mixing pattern is purely a phenomenological assumption [26], so is taking $U$ to be the maximal mixing pattern with a $\mathrm{CP}$-violating phase. Both of them might be able to result from certain flavor symmetries (e.g., discrete $A_{4}$ and $Z_{2}$ symmetries [27]). The third assumption is the near degeneracy between $M_{1}$ and $M_{2}$, or equivalently the smallness of $r$. It seems a bit contrived to take such tiny values of $r$, as shown in FIG. 1, to achieve the successful baryogenesis via leptogenesis. Why is $r$ so small? A few authors [28] have conjectured that $M_{1}$ and $M_{2}$ might be exactly degenerate at an energy scale higher than the seesaw scale (e.g., the GUT scale) and their small splitting at the seesaw scale is attributed to the radiative corrections.

- The minimal seesaw model with two heavy right-handed Majorana neutrinos $N_{1}$ and $N_{2}$ can be regarded as a limiting version of the conventional seesaw model with three right-handed Majorana neutrinos $N_{1}, N_{2}$ and $N_{3}$, in which $N_{3}$ is so heavy that it decouples from the theory at the very early stage. In order to gain the special mass spectrum (i.e., $M_{1} \approx M_{2} \ll M_{3}$ ), one possibility is to make use of the Froggatt-Nielsen (FN) mechanism and assign the charges of three right-handed Majorana neutrinos as $Q_{\mathrm{FN}}\left(N_{1 \mathrm{R}}\right)=-1, Q_{\mathrm{FN}}\left(N_{2 \mathrm{R}}\right)=+1$ and $Q_{\mathrm{FN}}\left(N_{3 \mathrm{R}}\right)=0$ [12]. Such a scenario allows $N_{1}$ and $N_{2}$ to be of $\mathcal{O}(1) \mathrm{TeV}$ and $N_{3}$ to be close to the GUT scale, and it can also accommodate the Yukawa couplings of $\mathcal{O}\left(10^{-7}\right)$. Besides flavor symmetries, the supersymmetry breaking may do the same job, as shown in Ref. [29].

- Radiative corrections to the light neutrino masses, the mixing angles and the CPviolating phases should also be taken into account, when those parameters run from the seesaw scale down to the electroweak scale. A generic analysis of the renormalizationgroup running effects in the minimal seesaw model has been done in Ref. [30]. Because $M_{1} \approx M_{2} \sim \mathcal{O}(1) \mathrm{TeV}$ is specified in our model, however, all of such running effects are expected to be negligibly small. Even in the supersymmetric case with large tan $\beta$, 
significant radiative corrections cannot emerge between the energy scales of $\mathcal{O}(1) \mathrm{TeV}$ and the electroweak scale.

- We stress that this $\mathrm{TeV}$-scale neutrino mass model is also viable in the framework of the minimal supersymmetric standard model. In particular, the above calculation of $\eta_{\mathrm{B}}$ can simply be extended to accommodate supersymmetry. The size of $\varepsilon_{i}$ in the supersymmetric case is twice as large as in the standard model, so is the magnitude

of $g_{*}$ [22]. The enhancement of $g_{*}$ gives rise to the same order suppression of the $\mathcal{O}\left(10^{-2}\right)$ dilution coefficient in Eq. (13) or Eq. (25), hence the estimate of $\eta_{\mathrm{B}}$ is not significantly changed by the introduction of supersymmetry. This observation, which has been confirmed by our detailed analysis, implies that the numerical results shown in FIG. 1 are expected to be roughly (order-of-magnitude) valid for the minimal supersymmetric standard model.

The upcoming neutrino oscillation experiments will test the predictions of our model for the neutrino mass spectrum, flavor mixing angles and $\mathrm{CP}$ violation. A more intriguing test of such seesaw-plus-leptogenesis models is certainly the direct search for TeV-scale righthanded Majorana neutrinos [32], which can be done at LHC and ILC in the future. Due to the tiny Yukawa couplings restricted by the models themselves, however, the observability of such new particles will be a big experimental challenge.

\section{Acknowledgments}

This work was supported in part by the National Natural Science Foundation of China.

[1] SNO Collaboration, Q.R. Ahmad et al., Phys. Rev. Lett. 89, 011301 (2002).

[2] For a review, see: C.K. Jung et al., Ann. Rev. Nucl. Part. Sci. 51, 451 (2001).

[3] KamLAND Collaboration, K. Eguchi et al., Phys. Rev. Lett. 90, 021802 (2003).

[4] K2K Collaboration, M.H. Ahn et al., Phys. Rev. Lett. 90, 041801 (2003).

[5] P. Frampton, S.L. Glashow, and T. Yanagida, Phys. Lett. B 548, 119 (2002). For a review with extensive references, see: W.L. Guo, Z.Z. Xing, and S. Zhou, Int. J. Mod. Phys. E 16, 1 (2007). 
[6] P. Minkowski, Phys. Lett. B 67, 421 (1977); T. Yanagida, in Proceedings of the Workshop on Unified Theory and the Baryon Number of the Universe, edited by O. Sawada and A. Sugamoto (KEK, Tsukuba, 1979), p. 95; M. Gell-Mann, P. Ramond, and R. Slansky, in Supergravity, edited by P. van Nieuwenhuizen and D. Freedman (North Holland, Amsterdam, 1979), p. 315; S.L. Glashow, in Quarks and Leptons, edited by M. Lévy et al. (Plenum, New York, 1980), p. 707; R.N. Mohapatra and G. Senjanovic, Phys. Rev. Lett. 44, 912 (1980).

[7] A.D. Sakharov, JETP Lett. 5, 24 (1967).

[8] V.A. Kuzmin, V.A. Rubakov, and M.E. Shaposhnikov, Phys. Lett. B 155, 36 (1985).

[9] M. Fukugita and T. Yanagida, Phys. Lett. B 174, 45 (1986).

[10] D.N. Spergel et al., astro-ph/0603449.

[11] M.R. Buckley and H. Murayama, hep-ph/0606088; F. del Aguila, J.A. Aguilar-Saavedra, and R. Pittau, hep-ph/0606198; and references therein.

[12] A. Pilaftsis and T.E.J. Underwood, Nucl. Phys. B 692, 303 (2004); and references therein.

[13] It seems possible to get around this difficulty by assuming a very contrived flavor structure in the resonant leptogenesis scenario. See, e.g., A. Pilaftsis, Phys. Rev. Lett. 95, 081602 (2005); A. Pilaftsis and T.E.J. Underwood, Phys. Rev. D 72, 113001 (2005).

[14] A. Anisimov, A. Broncano, and M. Plümacher, Nucl. Phys. B 737, 176 (2006).

[15] T. Endoh, S. Kaneko, S.K. Kang, T. Morozumi, and M. Tanimoto, Phys. Rev. Lett. 89, $231601(2002)$.

[16] W. Buchmüller and M. Plümacher, Phys. Lett. B 389, 73 (1996).

[17] P.F. Harrison, D.H. Perkins, and W.G. Scott, Phys. Lett. B 530, 167 (2002); Z.Z. Xing, Phys. Lett. B 533, 85 (2002); P.F. Harrison and W.G. Scott, Phys. Lett. B 535, 163 (2002); X.G. He and A. Zee, Phys. Lett. B 560, 87 (2003).

[18] See, e.g., A. Strumia and F. Vissani, hep-ph/0606054.

[19] W.M. Yao, et al., J. Phys. G 33, 1 (2006); H. Fritzsch and Z.Z. Xing, Phys. Lett. B 517, 363 (2001); Z.Z. Xing, Int. J. Mod. Phys. A 19, 1 (2004).

[20] S.P. Mikheyev and A.Yu. Smirnov, Sov. J. Nucl. Phys. 42, 913 (1985); L. Wolfenstein, Phys. Rev. D 17, 2369 (1978).

[21] C. Jarlskog, Phys. Rev. Lett. 55, 1039 (1985); D.D. Wu, Phys. Rev. D 33, 860 (1986).

[22] W. Buchmüller, P. Di Bari, and M. Plümacher, New J. Phys. 6, 105 (2004); G.F. Giudice, A. Notari, M. Raidal, A. Riotto, and A. Strumia, Nucl. Phys. B 685, 89 (2004). 
[23] S. Blanchet and P. Di Bari, hep-ph/0603107; hep-ph/0607330.

[24] R. Barbieri, P. Creminelli, A. Strumia, and N. Tetradis, Nucl. Phys. B 575, 61 (2000).

[25] A. Abada, S. Davidson, F.X. Josse-Michaux, M. Losada, and A. Riotto, JCAP 0604, 004 (2006); E. Nardi, Y. Nir, E. Roulet, and J. Racker, JHEP 0601, 164 (2006); A. Abada, S. Davidson, F.X. Josse-Michaux, M. Losada, and A. Riotto, hep-ph/0605281; S. Antusch, S.F. King, and A. Riotto, hep-ph/0609038; S. Pascoli and S.T. Petcov, hep-ph/0609125; G.C. Branco, R. Gonzalez Felipe, and F.R. Joaquim, hep-ph/0609297; S. Blanchet, P. Di Bari, G.G. Raffelt, hep-ph/0611337; S. Pascoli, S.T. Petcov and A. Riotto, hep-ph/0611338; A. De Simone and A. Riotto, hep-ph/0611357; S. Antusch, arXiv:0704.1591 [hep-ph].

[26] S. Chang, S.K. Kang, and K. Siyeon, Phys. Lett. B 597, 78 (2004); T. Kitabayashi, hep-ph/0703303.

[27] For a recent review with extensice references, see: R.N. Mohapatra and A.Yu. Smirnov, Ann. Rev. Nucl. Part. Sci. 56, 569 (2006).

[28] K. Turzynski, Phys. Lett. B 589, 135 (2004); R. Gonzale Felipe, F.R. Joaquim, and B.M. Nobre, Phys. Rev. D 70, 085009 (2004); G.C. Branco, R. Gonzalez Felipe, F.R. Joaquim, and B.M. Nobre, Phys. Lett. B 633, 336 (2006).

[29] T. Hambye, J. March-Russell and S.M. West, JHEP 07, 070 (2004).

[30] J.W. Mei and Z.Z. Xing, Phys. Rev. D 69, 073003 (2004).

[31] Here we do not discriminate one lepton flavor from another in the final states of $N_{i}$ decays. This flavor-independent leptogenesis scenario actually serves as a counter example to illustrate why flavor effects are important and must be taken into account in a $\mathrm{TeV}$-scale resonant leptogenesis model, as one can clearly see in section IV.

[32] The predictions for possible signatures of heavy right-handed Majorana neutrinos $N_{i}$ are strongly model-dependent. For example, they inevitably rely on the mixing strength between $N_{i}$ and three charged leptons. The latter has been constrained to some extent by current experimental data on the lepton universality and lepton-flavor-violating processes. See Ref. [11] for detailed discussions. 

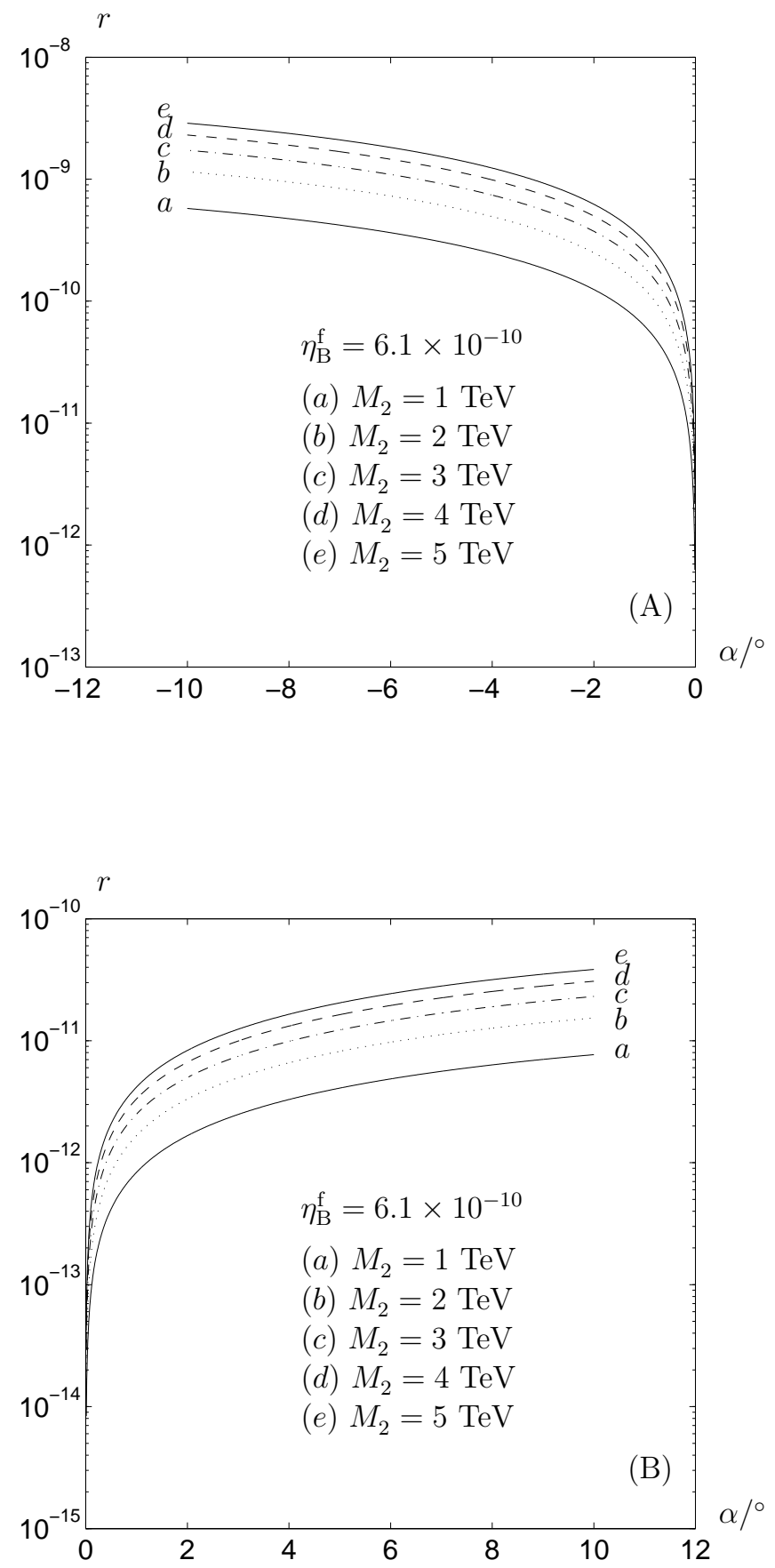

FIG. 1: Numerical illustration of the correlation between the mass splitting parameter $r$ and the CP-violating phase $\alpha$ to achieve the successful flavor-dependent resonant leptogenesis at the $\mathrm{TeV}$ scale: (A) in the $m_{1}=0$ case, and (B) in the $m_{3}=0$ case. 